Proceedings of the 2012 Winter Simulation Conference

C. Laroque, J. Himmelspach, R. Pasupathy, O. Rose, and A. M. Uhrmacher, eds.

\title{
EFFICIENT SIMULATION OF CHARGE TRANSPORT IN DEEP-TRAP MEDIA
}

\author{
Tim J. Brereton \\ Dirk P. Kroese \\ School of Mathematics and Physics \\ The University of Queensland \\ Brisbane 4072, AUSTRALIA
}

\author{
Ole Stenzel \\ Volker Schmidt \\ Institute of Stochastics \\ Ulm University \\ 89069 Ulm, GERMANY
}

\author{
Björn Baumeier \\ Max Planck Institute for Polymer Research \\ Ackermannweg 10 \\ 55128 Mainz, GERMANY
}

\begin{abstract}
This paper introduces a new approach to Monte Carlo estimation of the velocity of charge carriers driftdiffusing in a random medium. The random medium is modelled by a 1-dimensional lattice and the position of the charge carrier is modelled by a Markov jump process, whose state space is the set of lattice points. The transition rates of the Markov jump process are determined by the underlying energy landscape of the random medium. This energy landscape is modelled by a Gaussian process and contains regions of relatively low energy, in which charge carriers quickly become stuck. As a result, the state space is not adequately explored by the standard algorithms and the velocity of the charge carrier is poorly estimated. In addition, the conventional Monte Carlo estimators have very high variances. Our approach aims to reduce the number of simulation steps that are spent in the low energy problem regions. We do this by identifying the problem regions via a stochastic watershed algorithm. We then use a coarsened state space model, where the problem regions are treated as single states. In this way, we are able to simulate a semi-Markov process on the coarsened state space. This results in estimators that are unbiased and have considerably lower variance than the crude Monte Carlo alternatives.
\end{abstract}

\section{INTRODUCTION}

Electron transport in disordered organic semiconductors can be modeled by means of Markov jump processes on graphs, where the graph represents the random medium and the state of the jump process represents the position of the charge carrier. The transition (or hopping) rates of the jump process between adjacent vertices of the graph can be determined from the electrochemical and quantum mechanical properties of the semiconductor (Rühle et al. 2011). One of the main characteristics to be measured is the charge carrier mobility $\mu=v / F$, which is a function of the average velocity or drift velocity $v$ of the charge carrier as it passes through the random medium under the bias of an external electric field $F$. The drift velocity can be estimated via Monte Carlo simulation of the Markov jump process - called Kinetic Monte Carlo in the physics literature (Pasveer et al. 2005). Since the charge mobility influences the performance of a material in technological applications, e.g., the efficiency of organic solar cells, such a model for electron transport is a key ingredient of intensive efforts in in-silico design of high-efficiency organic semiconductors (Baumeier et al. 2012). The approach introduced by (Schönherr et al. 1981) and (Bässler 1993), which we call Crude Monte Carlo (CMC), has become a well-established method (Tessler et al. 2009) (van der Holst et al. 2011). A major problem, however, is that for a large variety of materials the energy landscape associated with the random 


\section{Brereton, Kroese, Stenzel, Schmidt, and Baumeier}

medium contains regions of low energy in which the charge carrier quickly becomes stuck. A consequence of this is that the estimation of drift velocity via crude simulation is not only very time-consuming, (often to the extent of being practically infeasible), but also leads to estimators that consistently overestimate the mobility. In addition, processes in organic electronics take place on multiple time and spatial scales. Therefore, it is essential to have computationally fast methods that analyze large system sizes over a long physical time without losing too much information about finer scale behaviour.

The purpose of this paper is to provide a fast alternative to the standard CMC algorithm that can be easily extended to 3-dimensional models that are of practical interest. We introduce a novel approach to the problem of deep energy traps, where we identify the problem areas using a stochastic watershed algorithm. We then construct a coarsened state space model, under which the problem regions can be traversed in a single step of the simulation. In the literature, this type of problems has been discussed in the context of e.g. nearly decomposable Markov processes (Courtois 1977, Simon and Ando 1961) and multiple time-scale Markov processes (Evans 1996, Tse et al. 1995). A similar strategy of coarsening the state space is used in (Somoza and Ortuño 2005) to study the relaxation of Coulomb glasses at low temperature. However, the latter approach only considers pairs of problem states, and does not segment the state space prior to the simulation. In the present paper, we focus on a 1-dimensional system, which already describes the main physical processes quite well (van der Holst et al. 2011). However, the basic method presented in this paper can be extended to 3-dimensional models. In particular, the stochastic watershed segmentation can be directly applied in 2D and 3D.

\section{MODEL}

In this section, the random medium is modelled by a 1-dimensional lattice and the position of the charge carrier is modelled by a Markov jump process, whose state space is the set of lattice points. The transition rates of the Markov jump process are determined by the underlying energy landscape of the random medium. Such a model is related to both 1-dimensional Gaussian Disorder Models (GDM) and Correlated Disorder Models (CDM) (van der Holst et al. 2011). In this paper, we consider a correlated energy landscape, as it is more accurate for small molecule systems.

\subsection{Electron Transfer Rates}

Electron transfer rates between neighboring molecules $i$ and $j$ in disordered organic semiconductors can be obtained from the high-temperature limit of classical charge-transfer theory (Marcus 1993) according to

$$
q_{i j}=\frac{2 \pi}{\hbar} \frac{J_{i j}^{2}}{\sqrt{4 \pi \lambda_{i j} k_{\mathrm{B}} T}} \exp \left[-\frac{\left(\Delta E_{i j}-\lambda_{i j}\right)^{2}}{4 \lambda_{i j} k_{\mathrm{B}} T}\right],
$$

where $\hbar=6.58 \cdot 10^{-16} \mathrm{eV} \cdot \mathrm{s}$ is the reduced Planck constant, $k_{\mathrm{B}}=8.62 \cdot 10^{-5} \mathrm{eV} \cdot \mathrm{K}^{-1}$ is Boltzmann's constant, and $T$ the temperature. In realistic morphologies, the quantities $J_{i j}$ (transfer integral), $\lambda_{i j}$ (reorganization energy) and $\Delta E_{i j}$ (driving force) are evaluated explicitly using a combination of quantummechanical electronic structure techniques and classical simulation methods, see (Rühle et al. 2011). The driving force $\Delta E_{i j}=E_{i}-E_{j}+e\left(x_{j}-x_{i}\right) F$ comprises static site energy differences $E_{i}-E_{j}$ and a drift term $e\left(x_{i}-x_{j}\right) F$ due to an externally applied electric field $F$ acting on the elementary charge $e$, where $x_{i}$ is the Cartesian coordinate of the center of mass of molecule $i$.

\subsection{Energy Landscape}

In the approaches described in (Coropceanu et al. 2007), (Rühle et al. 2011) and references therein, the motion of the charge carrier is modelled as a time-homogeneous Markov jump process $\left\{X_{t}\right\}_{t \geqslant 0}$ where the state space is the vertex set of a 3-dimensional graph. In the following, we consider a 1-dimensional abstraction of such 3 -dimensional models. We use a finite state space $\{1, \ldots, n\}$, imposing cyclic boundary 


\section{Brereton, Kroese, Stenzel, Schmidt, and Baumeier}

conditions to approximate an infinite random medium. One of the main factors determining the dynamics of the jump process via eq. 1 is the underlying energy landscape which determines the transition rates $q_{i j}$. We consider jumps to nearest neighbouring states only, i.e., we put $q_{i j}=0$ if $|i-j| \neq 1$. In amorphous tris-(8-hydroxyquinoline)aluminum, a typical organic semiconductor, a microscopic evaluation based on a simulated morphology yield energies $E_{i}$ which are positively correlated across neighboring molecules (see (Rühle et al. 2011)), i.e., the energy landscape, $E_{1}, E_{2}, \ldots, E_{n}$, behaves like a moving average process. More precisely, $E_{i}$ satisfies

$$
E_{i}=\sum_{k=-K}^{K} \sqrt{\frac{\omega}{2 K+1}} \varepsilon_{i-k}+\sqrt{1-\omega} \widetilde{\varepsilon}_{i} \quad, \quad i=1, \ldots, 100 \bmod n,
$$

where the $\left\{\varepsilon_{i}\right\}$ and $\left\{\widetilde{\varepsilon}_{i}\right\}$ are sequences of independent and identically distributed $\mathrm{N}\left(m, \sigma^{2}\right)$ random variables. Note that $K$ controls the broadness of the spatial correlation of $E_{i}$, and $\omega$ controls the local roughness of the energy landscape; in particular, for $\omega=0$ all $\left\{E_{i}\right\}$ are independent and the energy landscape becomes very rough. A typical realization with a parametrization of the microscopic data from (Rühle et al. 2011), i.e., $n=100, K=1, m=-0.182 \mathrm{eV}, \sigma^{2}=0.034 \mathrm{eV}^{2}$, and $\omega=0.9$, is given in Figure 1 .

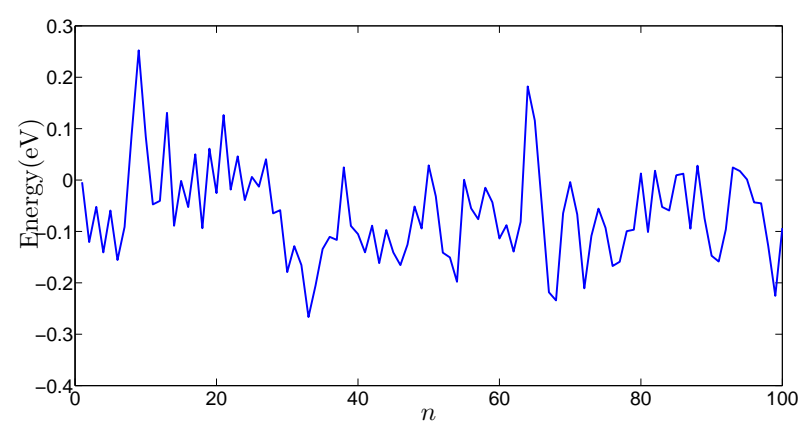

Figure 1: Moving average energy landscape.

All remaining parameters to determine the transition rates $q_{i j}$ in eq. 1 except $\Delta E_{i j}$ are chosen constant. More precisely, $J_{i j}^{2}=1.7 \cdot 10^{-4} \mathrm{eV}^{2}$ (an average value of the microscopic data, assuming that neighboring sites are separated by $1 \mathrm{~nm}), \lambda_{i j}=0.23 \mathrm{eV}$, and $T$ is set to room temperature so that $k_{\mathrm{B}} T=0.025 \mathrm{eV}$. The energy difference is given by $\Delta E_{i j}=E_{i}-E_{j}+(j-i) \delta$, where $\delta=0.01 \mathrm{eV}$ is the drift term, corresponding to an external electric field $F=10^{7} \mathrm{~V} / \mathrm{m}$. In general, the charge carrier is more likely to move from a higher energy region to a lower energy region than it is to move from a lower energy region to a higher energy region. Thus, it tends to spend a large amount of time in deep energy valleys.

\subsection{Drift Velocity}

The drift velocity $v$ is defined mathematically as the long-run average velocity with which a charge carrier travels through the random medium:

$$
v=\lim _{t \rightarrow \infty} \frac{X_{t}-X_{0}}{t}
$$

This quantity is usually estimated via Monte Carlo methods. For convenience, in the 1-dimensional case we can treat the Markov jump process (MJP) as a birth-death process, with birth rates $\alpha_{j}=q_{j, j+1}$ and death rates $\beta_{j}=q_{j, j-1}$. We then simulate a MJP, tracking $d$, the distance travelled to the right by the jump process. In the presented algorithms, we focus on $N$ rather than $t$ in order to highlight how the accuracy of the estimator changes as a function of step size. The CMC approach to the estimation of drift velocity is given in the following algorithm: 
Algorithm 2.1 (Crude Monte Carlo estimation of drift velocity) For a given number of steps $N$

1. Set $d=0, j=0$ and $t=0$.

2. Draw $X_{0}$ uniformly from $\{1, \ldots, n\}$.

3. With probability $\frac{\alpha_{X_{j}}}{\alpha_{X_{j}}+\beta_{X_{j}}}$, set $X_{j+1}=X_{j}+1$ and $d=d+1$. Otherwise, set $X_{j+1}=X_{j}-1$ and $d=d-1$.

4. Draw $\tau_{j} \sim \operatorname{Exp}\left(\alpha_{X_{j}}+\beta_{X_{j}}\right)$ and set $t=t+\tau_{j}$.

5. Set $j=j+1$. If $j<N$, repeat from Step 3 .

6. Return $\widehat{v}=d / t$.

Using the CMC approach, the drift velocity is significantly overestimated for smaller values of $N$. The addition of a burn-in period reduces, but does not remove, the bias. Figure 2 shows average point estimates of $\widehat{v}$ for the CMC estimator and the CMC estimator with a burn-in period that is $10 \%$ of the sample size $N$.

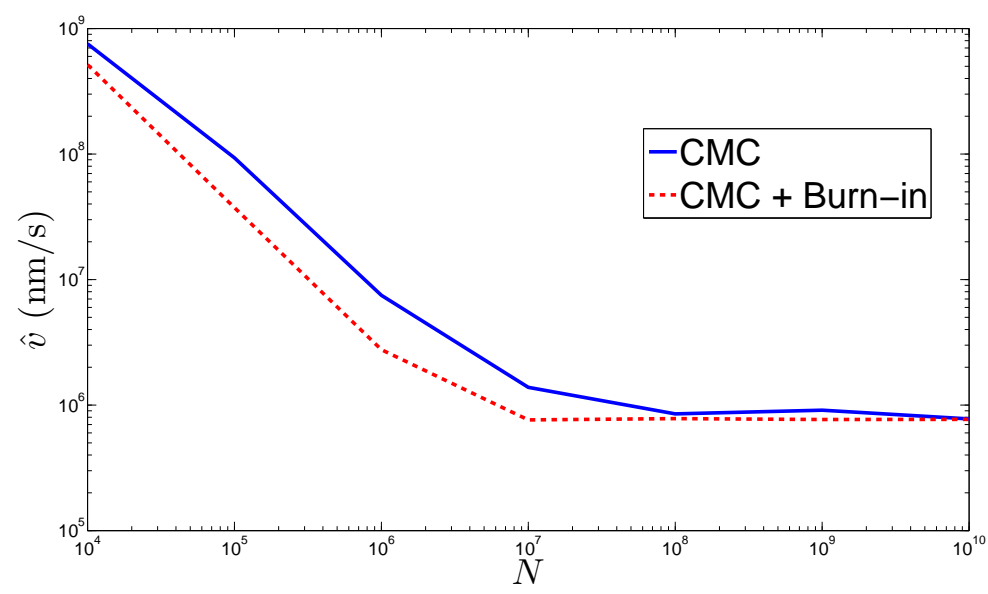

Figure 2: Estimates of drift velocity vs. sample size

In order to assess the performance of the estimators, we estimated their mean and standard deviation via a bootstrap. The results are summarized in Table 1. Note that the variance of the estimator $\widehat{v}$ is very large. In particular, for smaller values of $N$ the estimated standard deviation is of the same order as the estimated mean of $\widehat{v}$.

Table 1: Mean and standard deviation of $\widehat{v}$ (in $\mathrm{nm} / \mathrm{s}$ ) using CMC with a burn-in period

\begin{tabular}{ccc}
\hline$N$ & Mean & Standard deviation \\
\hline $10^{4}$ & $5.15 \times 10^{8}$ & $6.85 \times 10^{8}$ \\
$10^{5}$ & $3.74 \times 10^{7}$ & $6.47 \times 10^{7}$ \\
$10^{6}$ & $2.76 \times 10^{6}$ & $5.19 \times 10^{6}$ \\
$10^{7}$ & $7.62 \times 10^{5}$ & $1.13 \times 10^{6}$ \\
$10^{8}$ & $7.80 \times 10^{5}$ & $3.65 \times 10^{5}$ \\
$10^{9}$ & $7.68 \times 10^{5}$ & $9.87 \times 10^{4}$ \\
$10^{10}$ & $7.70 \times 10^{5}$ & $1.35 \times 10^{4}$ \\
& & \\
\hline
\end{tabular}


The primary obstacle to efficient estimation is the existence of problem regions, where the MJP becomes trapped for very long periods of time, commonly up to $10^{6}$ steps. As a result, the state space is not adequately explored by the standard algorithm (for a finite state space, with cyclic boundary conditions, the MJP does not come close to the stationary distribution), and the time spent in problem regions is often underestimated.

\section{FAST SIMULATION OF CHARGE TRANSPORT}

The idea behind our approach to improving the estimation of the drift velocity is to coarsen the state space, so that the problem regions can be traversed in a single step. This is done by

1. identifying the problem regions via a stochastic watershed algorithm,

2. reducing these problem regions to single states,

3. replacing the MJP with a semi-Markov process,

4. carrying out the simulation with the new coarsened state space model.

\subsection{Stochastic Watershed Algorithm}

In order to increase the efficiency of the simulations, we need to coarsen the state-space in such a way that charge carrier moves between each pair of neighboring states with sufficiently high transition rates. This is done by collapsing the 'valleys' in the energy landscape into single (super-) states. In order to do this, it is necessary to identify the relatively low energy regions of the energy landscape. The approach we use is a modification of the standard watershed transformation considered in image segmentation, see e.g. (Beucher and Meyer 1993). The stochastic watershed is chosen since it directly extends to 2D and 3D models and can handle large system sizes. Firstly, for segmentation purposes, we create an adjusted energy landscape which takes into account the drift of the MJP. This is because the MJP with drift corresponds to a MJP with zero-drift when the energy landscape is tilted appropriately; see Figure 3. More precisely, the energy landscape $E_{1}, E_{2}, \ldots, E_{n}$ is transformed to $\widetilde{E}_{1}, \widetilde{E}_{2}, \ldots, \widetilde{E}_{n}$ with

$$
\widetilde{E}_{i}=E_{i}-i \delta, \quad i=1, \ldots, n .
$$

Note that cyclic boundary conditions are applied to the tilted energy landscape, i.e., the energy difference between states $n$ and 1 is shifted by $n \delta$.

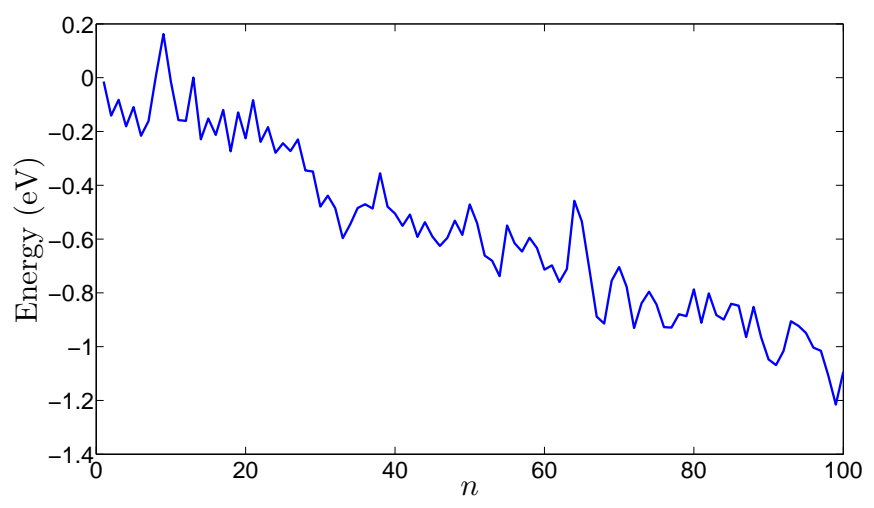

Figure 3: Tilted energy landscape.

The problem regions are determined by the tilted energy landscape, since the transition rates $q_{i j}$ only depend on the energy differences for the tilted energy landscape. Interpreting the tilted energy landscape as a 1D image, the coarsening of the state space can be viewed as the problem of image segmentation 


\section{Brereton, Kroese, Stenzel, Schmidt, and Baumeier}

in image analysis. A widely applied tool in image segmentation is the watershed transformation. Its key idea was introduced by (Beucher and Lantuéjoul 1979). Initially developed for gray-scale images, the watershed transformation was extended for continuous values, see (Najman and Schmitt 1994). The algorithm normally used today was developed by (Beucher and Meyer 1993). The basic idea of the watershed algorithm is to consider the image as a topographic relief. This topographic relief is then flooded with water starting at all local minima. If water from different sources (local minima) merge at a certain point, a watershed marker is set. The set of watershed markers segment the image into disjoint regions, so-called basins; see Figure 4 for a schematic example.

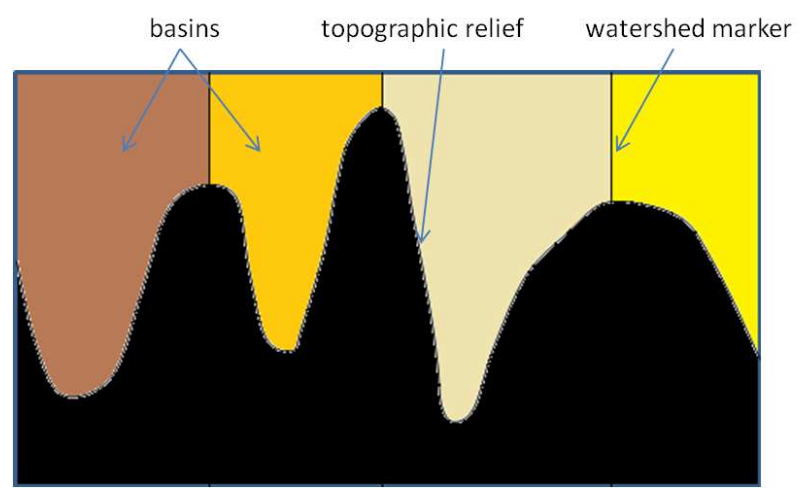

Figure 4: Watershed transformation.

A limitation of the standard watershed transformation is that it often yields oversegmentation. That is, the segmentation into basins (here: super-states of the jump process to be constructed) is too fine. We therefore use the stochastic watershed transformation introduced by (Angulo and Jeulin 2007). The idea is to replace the local minima as starting points of flooding by random starting points generated by a homogeneous Poisson process with some intensity $\lambda$, here discretized on the discrete 1D lattice that forms the state space. The outcome is a random set of watershed markers separating the (random) basins. The procedure is repeated an appropriate number of times (here: 6000 times, in the case of a energy landscape with length $n=100$ ) and the relative frequencies of markers on the discrete lattice are computed. Finally, a global thresholding is performed and markers are only accepted if their relative frequencies exceed a certain threshold $T$. In our case, a homogeneous 1D Poisson process with intensity $\lambda=0.1$ is chosen, together with $T=0.1$. The choices of $\lambda$ and $T$ influence the segmentation of the energy landscape. In general, $\lambda$ is chosen so that the expected number of starting points is less than the total number of local minima. The threshold $T$ determines the size of the super-states. A higher threshold yields larger super-states. The threshold $T$ is chosen such that the size of the super-states is numerically feasible. For small system sizes, it is also possible to collapse all states into a single super-state. The result of the stochastic watershed segmentation with the above mentioned parameters is displayed in Figure 5. 

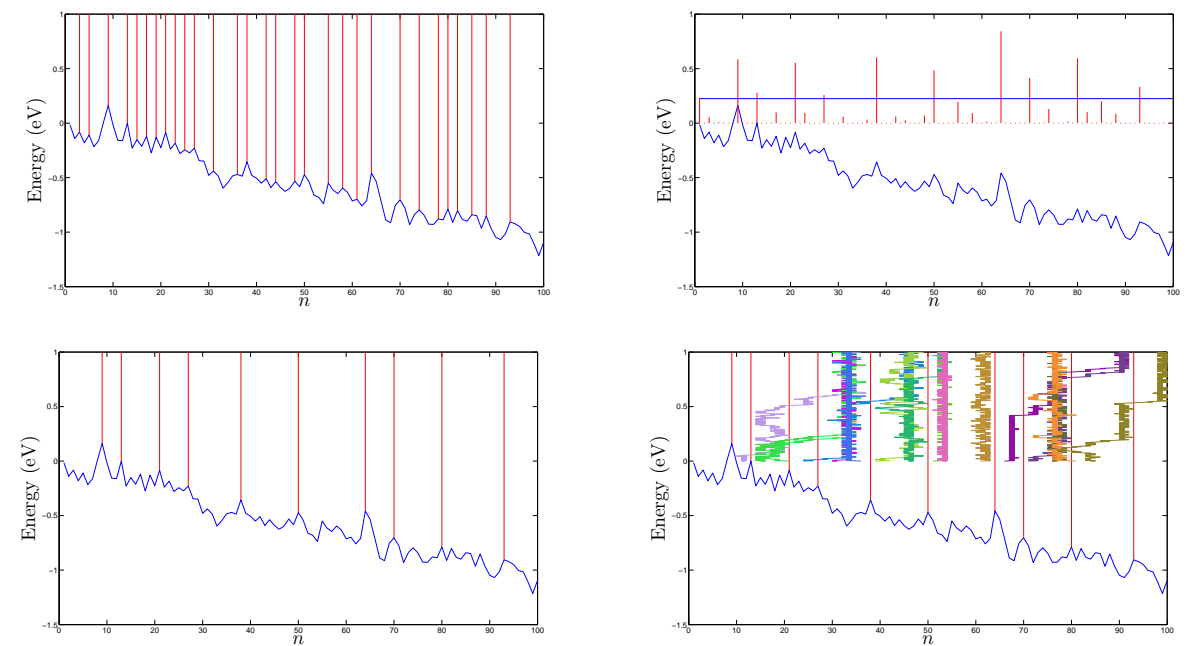

Figure 5: Top. Left: standard watershed segmentation of the tilted energy landscape. Right: relative frequencies of watershed markers computed from the stochastic watershed (red) together with the threshold $T=0.225$ (blue line). Bottom. Left: watershed segmentation by the stochastic watershed. Right: watershed segmentation by the stochastic watershed together with samples of the MJP for different starting points.

\subsection{Coarsened State Space Model}

The stochastic watershed segmentation allows us to decompose the state space $\{1, \ldots, n\}$ into a random number of subsets. They are separated from each other by the points $S_{1}, \ldots, S_{k} \in\{1, \ldots, n\}$ at which the relative frequencies of watershed markers exceed the threshold $T$. Each of these segmentation points consists of a single state of the original MJP. We amalgamate the collection of states between any two consecutive segmentation points into a single super-state. Thus, we construct the coarsened state space $\{\tilde{1}, \ldots, \tilde{n}\}$, where each state is either a segmentation point or a problem region. A schematic example of this is shown in Figure 6.
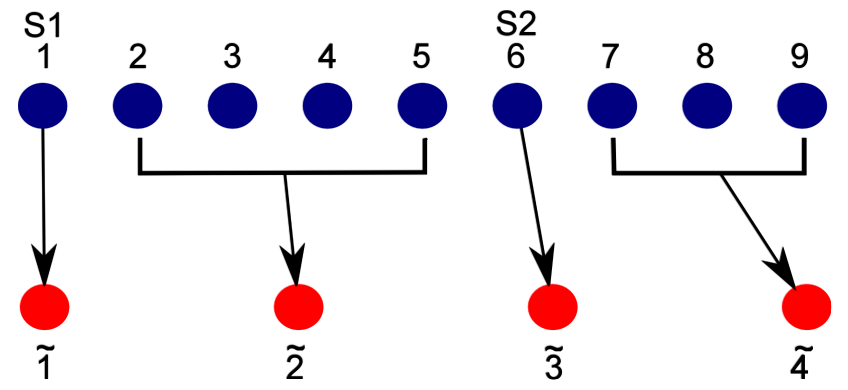

Figure 6: Coarsened state space.

Given the coarsened state space, we can describe the movement of the charge carrier by a semi-Markov process $\left\{\tilde{X}_{t}\right\}_{t \geqslant 0}$; see e.g. (Limnios and Oprisan 2001). The jump chain is still Markovian and, while the sojourn times are no longer exponentially distributed, they remain conditionally independent.

In order to calculate the charge carrier mobility, we need to track the distance traveled by the charge carrier and the time taken to traverse this distance. Because we are considering a long-run quantity, we can replace the random time spent in a super-state with its expected value. Thus, for each super-state or segmentation point, $i=\tilde{1}, \ldots, \tilde{n}$, we calculate 9 values. The first value, $d_{i}$, represents the distance 


\section{Brereton, Kroese, Stenzel, Schmidt, and Baumeier}

travelled if the entire state is traversed. If the state is entered from the left, the charge carrier moves left with probability $P_{i}^{\mathrm{LL}}$ and moves right with probability $P_{i}^{\mathrm{LR}}$. The mean time taken to move left is denoted by $\tau_{i}^{\mathrm{LL}}$, and the mean time taken to move right by $\tau_{i}^{\mathrm{LR}}$. If the state is entered from the right, the analagous quantities are denoted by $P_{i}^{\mathrm{RL}}, P_{i}^{\mathrm{RR}}, \tau_{i}^{\mathrm{RL}}$, and $\tau_{i}^{\mathrm{RR}}$. These quantities are determined by considering a Markov jump process on the states within the super-state, with the two adjoining segmentation points acting as absorbing barriers. To calculate the probabilities $P_{i}^{\mathrm{LL}}, P_{i}^{\mathrm{LR}}, P_{i}^{\mathrm{RL}}, P_{i}^{\mathrm{RR}}$, we partition the state space of this MJP such that the transition matrix $Q$ and jump matrix $J$ take the following forms

$$
Q=\left(\begin{array}{cc}
0 & 0 \\
S_{0} & S
\end{array}\right) \quad \text { and } \quad J=\left(\begin{array}{cc}
I & 0 \\
R & T
\end{array}\right)
$$

respectively. The matrix $P=\left(p_{j k}\right)$ of absorption probabilities from transient state $j$ into absorbing state $k$ is given by $P=(I-T)^{-1} R$. We set $P_{i}^{\mathrm{LL}}=p_{1,1}, P_{i}^{\mathrm{LR}}=p_{1,2}, P_{i}^{\mathrm{RL}}=p_{r-l-1,1}$ and $P_{i}^{\mathrm{LL}}=p_{r-l-1,2}$. To find the expected times until absorption, we note that the matrix of the densities of the absorption times, $\left(f_{j, k}(t)\right)$, from transient state $j$ into absorbing state $k$ is given by $\mathrm{e}^{t S} S_{0}$, where $\mathrm{e}^{t S}$ is a matrix exponential. If we set $f_{j, k}^{\tau}(t)=f_{j k}(t) / p_{j, k}$, we obtain the densities of the conditional absorption times. The matrix of expected absorption times, $\left(\mathbb{E} t_{j, k}\right)$, is given by $\left(S^{2}\right)^{-1} S_{0}$. Thus, the expected conditional absorption times are given by $\tau_{j, k}=\mathbb{E} t_{i j} / p_{j, k}$. Hence, we can calculate $\tau_{i}^{\mathrm{LL}}=\tau_{1,1}, \tau_{i}^{\mathrm{LR}}=\tau_{1,2}, \tau_{i}^{\mathrm{RL}}=\tau_{r-l-1,1}$ and $\tau_{i}^{\mathrm{RR}}=\tau_{r-l-1,2}$.

\subsection{Aggregate Simulation Algorithm}

The procedure described above, which is based on the aggregation of critical regions of states into single super-states, can be summarized as follows.

\section{Algorithm 3.1 (Aggregate Monte Carlo Estimation of Drift Velocity)}

Let $N$ be the number of steps

1. Given an initial energy landscape $E_{1}, \ldots, E_{n}$, identify an appropriate coarsened state space $\tilde{1}, \ldots, \tilde{n}$, via the stochastic watershed algorithm.

2. As described above, calculate the absorption probabilities and expected sojourn times corresponding to the new (aggregate) state space.

3. Set $d=0, j=0$ and $t=0$.

4. Draw $X_{0}$ uniformly from $\{\tilde{1}, \ldots, \tilde{n}\}$ and the state variable LEFT uniformly from \{true, false\}.

5. If LEFT $=$ true, then with probability $p_{X_{j}}^{\mathrm{LL}}$ set $X_{j+1}=X_{j}-1, d=d-1$, LEFT $=$ false and $t=t+\tau_{X_{j}}^{\mathrm{LL}}$; otherwise, set $X_{j+1}=X_{j}+1, d=d+d_{X_{j}}$ and $t=t+\tau_{X_{j}}^{\mathrm{LR}}$.

6. If LEFT $=$ false, then with probability $p_{X_{j}}^{\mathrm{RL}}$ set $X_{j+1}=X_{j}-1, d=d-d_{X_{j}}$ and $t=t+\tau_{X_{j}}^{\mathrm{RL}}$; otherwise, set $X_{i+1}=X_{j}+1, d=d+1$, LEFT $=$ true and $t=t+\tau_{X_{j}}^{\mathrm{RR}}$.

7. Set $j=j+1$. If $j<N$, repeat from Step 5 .

8. Return $\widehat{v}=d / t$.

Figure 7 shows the performance of the aggregate Monte Carlo estimator relative to the CMC and CMC with burn-in estimators. 
Brereton, Kroese, Stenzel, Schmidt, and Baumeier

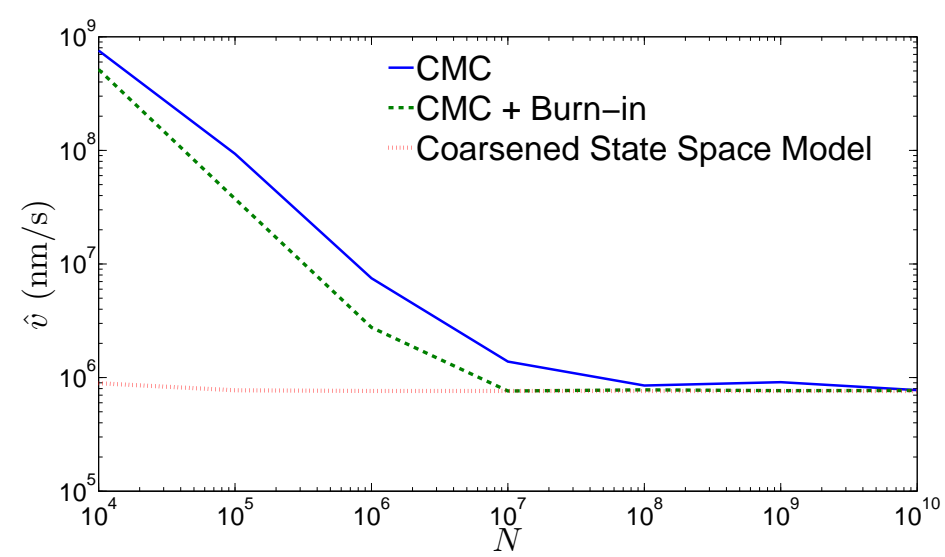

Figure 7: Estimates of drift velocity vs. sample size.

\section{NUMERICAL RESULTS}

In order to compare our approach of Aggregate Monte Carlo (AMC) simulation with the conventional Monte Carlo approaches stated in Section 2.3, we fixed a number of energy landscapes and estimated the drift velocity using both the CMC and AMC estimators. The means and standard deviations of the estimators were calculated using a bootstrap. The obtained numerical results are summarized in Table 2-4, where Figure 7 corresponds to Table 2. Note that in all cases the CMC estimator is considerably overbiased for small sample sizes and that the standard deviation of the AMC estimator is several orders of magnitude lower than that of the CMC estimator. In particular, these findings do not depend on the sizes $n=100$, 1000, and 10000 of the considered energy landscapes. Note however that the values of the means and standard deviations of $\widehat{v}$ vary from case to case. The reason for this is the randomness of the energy landscape, being modelled by a Gaussian process. In other words, the values presented in Tables 2, 3, and 4 correspond to estimates for conditional drift velocities, given three different samples drawn from the Gaussian energy landscape.

Table 2: Means and standard deviations (sd) of $\widehat{v}$ (in $\mathrm{nm} / \mathrm{s}$ ) for CMC with burn-in and AMC, for a landscape of size $n=100$.

\begin{tabular}{ccccc}
\hline$N$ & CMC mean & CMC sd & AMC mean & AMC sd \\
\hline $10^{4}$ & $5.15 \times 10^{8}$ & $6.85 \times 10^{8}$ & $8.92 \times 10^{5}$ & $5.20 \times 10^{5}$ \\
$10^{5}$ & $3.74 \times 10^{7}$ & $6.47 \times 10^{7}$ & $7.73 \times 10^{5}$ & $1.62 \times 10^{5}$ \\
$10^{6}$ & $2.76 \times 10^{6}$ & $5.19 \times 10^{6}$ & $7.62 \times 10^{5}$ & $5.16 \times 10^{4}$ \\
$10^{7}$ & $7.62 \times 10^{5}$ & $1.13 \times 10^{6}$ & $7.62 \times 10^{5}$ & $1.62 \times 10^{4}$ \\
$10^{8}$ & $7.80 \times 10^{5}$ & $3.65 \times 10^{5}$ & $7.61 \times 10^{5}$ & $5.36 \times 10^{3}$ \\
$10^{9}$ & $7.68 \times 10^{5}$ & $9.87 \times 10^{4}$ & $7.61 \times 10^{5}$ & $1.63 \times 10^{3}$ \\
$10^{10}$ & $7.70 \times 10^{5}$ & $1.35 \times 10^{4}$ & $7.61 \times 10^{5}$ & $4.77 \times 10^{2}$ \\
\hline
\end{tabular}

\section{CONCLUSIONS AND FURTHER RESEARCH}

We introduced a new approach to Monte Carlo estimation of the velocity of charge carriers drift-diffusing in a random medium, where the random medium is modelled by a 1-dimensional lattice and the position of the charge carrier is modelled by a Markov jump process, whose state space is the set of lattice points. 


\section{Brereton, Kroese, Stenzel, Schmidt, and Baumeier}

Table 3: Means and standard deviations (sd) of $\widehat{v}$ (in $\mathrm{nm} / \mathrm{s}$ ) for CMC with burn-in and AMC, for a landscape of size $n=1000$.

\begin{tabular}{ccccc}
\hline$N$ & CMC mean & CMC sd & AMC mean & AMC sd \\
\hline $10^{4}$ & $1.26 \times 10^{9}$ & $1.58 \times 10^{9}$ & $1.76 \times 10^{8}$ & $2.82 \times 10^{7}$ \\
$10^{5}$ & $4.50 \times 10^{8}$ & $3.96 \times 10^{8}$ & $1.73 \times 10^{8}$ & $8.60 \times 10^{6}$ \\
$10^{6}$ & $1.95 \times 10^{8}$ & $1.02 \times 10^{8}$ & $1.72 \times 10^{8}$ & $2.70 \times 10^{6}$ \\
$10^{7}$ & $2.03 \times 10^{8}$ & $3.32 \times 10^{7}$ & $1.72 \times 10^{8}$ & $8.46 \times 10^{5}$ \\
$10^{8}$ & $2.04 \times 10^{8}$ & $9.66 \times 10^{6}$ & $1.72 \times 10^{8}$ & $2.65 \times 10^{5}$ \\
\hline
\end{tabular}

Table 4: Means and standard deviations (sd) of $\widehat{v}$ (in $\mathrm{nm} / \mathrm{s}$ ) for CMC with burn-in and AMC, for a landscape of size $n=10000$.

\begin{tabular}{ccccc}
\hline$N$ & CMC mean & CMC sd & AMC mean & AMC sd \\
\hline $10^{4}$ & $8.97 \times 10^{9}$ & $1.12 \times 10^{9}$ & $6.66 \times 10^{7}$ & $5.36 \times 10^{7}$ \\
$10^{5}$ & $3.32 \times 10^{8}$ & $3.85 \times 10^{8}$ & $1.35 \times 10^{7}$ & $7.86 \times 10^{6}$ \\
$10^{6}$ & $1.33 \times 10^{8}$ & $1.02 \times 10^{8}$ & $1.03 \times 10^{7}$ & $1.76 \times 10^{6}$ \\
$10^{7}$ & $4.86 \times 10^{7}$ & $2.92 \times 10^{7}$ & $1.01 \times 10^{7}$ & $5.60 \times 10^{5}$ \\
$10^{8}$ & $1.12 \times 10^{7}$ & $1.31 \times 10^{6}$ & $1.00 \times 10^{7}$ & $1.68 \times 10^{5}$ \\
\hline
\end{tabular}

The transition rates of the Markov jump process are determined by the underlying energy landscape of the random medium. This energy landscape is modelled by a Gaussian process and contains regions of relatively low energy, in which charge carriers quickly become stuck. As a result, the state space is not adequately explored by the standard algorithms and the velocity of the charge carrier is poorly estimated. In addition, the conventional Monte Carlo estimators have very high variances. Our approach aims to reduce the number of simulation steps that are spent in the low energy problem regions. We do this by identifying the problem regions via a stochastic watershed algorithm. We then use a coarsened state space model, where we aggregate the problem regions to a single (super-) state. We are then able to simulate a semi-Markov process on the aggregate state space. This results in estimators that are unbiased and have considerably lower variance than the crude Monte Carlo estimators.

This paper has focused on the improved estimation of drift velocity in a $1 \mathrm{D}$ random medium. As an immediate extension of this work, we aim to extend the use of the AMC approach to 2 and 3-dimensional models. The methods described in this paper, such as the stochastic watershed algorithm, can all be applied in a higher dimensional setting. In addition, we aim to investigate whether the Markov Jump Process modelling the charge carrier's position can be profitably replaced by a diffusion approximation.

\section{Acknowledgements}

This work was partially supported by the DAAD / Go8 Australia-Germany Joint Research Cooperation Scheme and Deutsche Forschungsgemeinschaft (DFG) under the Priority Program "Elementary Processes of Organic Photovoltaics" (SPP 1355). Dirk Kroese acknowledges the support of the Australian Research Council under grant number DP0985177. We are grateful to Hans Daduna for helpful comments and Falk May for a critical reading of the manuscript. 
Brereton, Kroese, Stenzel, Schmidt, and Baumeier

\section{REFERENCES}

Angulo, J., and D. Jeulin. 2007. "Stochastic watershed segmentation". In 8th International Symposium on Mathematical Morphology, 265-276.

Bässler, H. 1993. "Charge transport in disordered organic photoconductors". Physica Status Solidi (b) 175 (15): $15-56$.

Baumeier, B., F. May, C. Lennartz, and D. Andrienko. 2012. "Challenges for in silico design of organic semiconductors". Journal of Materials Chemistry 22:10971-10976.

Beucher, S., and C. Lantuéjoul. 1979. "Use of watersheds in contour detection". In International workshop on image processing, real-time edge and motion detection / estimation.

Beucher, S., and F. Meyer. 1993. "The morphological approach to segmentation: the watershed transformation". In Mathematical Morphology in Image Processing, Volume 34, 433-481.

Coropceanu, V., J. Cornil, D. A. da Silva Filho, Y. Olivier, R. Silbey, and J. Brédas. 2007, April. "Charge transport in organic semiconductors". Chemical Reviews 107 (4): 926-952.

Courtois, P. 1977. Decompsability. ACM Monograph Series. New York: Academic Press.

Evans, S. 1996. "Analysing system behaviour on different time scales". In Stochastic Networks - Theory and Applications, edited by F. Kelly, S. Zachary, and I. Ziedins, Volume 4 of Royal Statistical Society Lecture Notes Series, Chapter 14, 231-246. Oxford: Clarendon Press.

Limnios, N., and G. Oprisan. 2001. Semi-Markov processes and reliability. Series Statistics for Industry and Technology. Boston: Birkhäuser.

Marcus, R. A. 1993. "Electron transfer reactions in chemistry. Theory and experiment". Reviews of Modern Physics 65 (3): 599.

Najman, L., and M. Schmitt. 1994. "Watershed of a continuous function". In Signal Processing (Special issue on Mathematical Morphology.), Volume 38, 99-112.

Pasveer, W., J. Cottaar, C. Tanase, R. Coehoorn, P. Bobbert, P. Blom, D. de Leeuw, and M. Michels. 2005. "Unified description of charge-carrier mobilities in disordered semiconducting polymers". Physical Review Letters 94:206601-1-206601-4.

Rühle, V., A. Lukyanov, F. May, M. Schrader, T. Vehoff, J. Kirkpatrick, B. Baumeier, and D. Andrienko. 2011. "Microscopic simulations of charge transport in disordered organic semiconductors". Journal of Chemicyl Theory and Computation 7 (10): 3335-3345.

Schönherr, G., H. Bässler, and M. Silver. 1981. "Dispersive hopping transport via sites having a Gaussian distribution of energies". Philosophical Magazine B 44 (1): 47-61.

Simon, H., and A. Ando. 1961. "Aggregation of variables in dynamic systems". Econometrica 29 (2): $111-138$.

Somoza, A., and M. Ortuño. 2005. "Monte Carlo method for relaxation in electron glasses.". Physical Review $B 72$.

Tessler, N., Y. Preezant, N. Rappaport, and Y. Roichman. 2009. "Charge transport in disordered organic materials and its relevance to thin-film devices: a tutorial review". Advanced Materials 21:2741-4761.

Tse, D., R. Gallager, and J. Tsitsiklis. 1995. "Statistical multiplexing of multiple time-scale Markov streams". IEEE J. Sel. Areas Commun. 13 (6): 1028-1038.

van der Holst, J., F. van Oost, R. Coehoorn, and P. Bobbert. 2011. "Monte Carlo study of charge transport in organic sandwich-type single-carrier devices: effects of coulomb interactions". Physical Review B 83:085206-1 - 085206-13.

\section{AUTHOR BIOGRAPHIES}

Tim Brereton is a PhD student at the University of Queensland. He has a Bachelor of Science (Honours) in Mathematics and a Masters of International Economics and Finance, both from the University of Queensland. His research interests include simulation, computational statistics and mathematical finance. 


\section{Brereton, Kroese, Stenzel, Schmidt, and Baumeier}

His email address is tim.brereton@uqconnect.edu.au.

Ole Stenzel is a $\mathrm{PhD}$ student at the Faculty of Mathematics and Economics of Ulm University. He has a Master (Diploma) in Mathematics from the University of Hamburg. His research interests include stochastic geometry, spatial statistics, and Monte Carlo simulation of spatial stochastic models as well as their applications to 3D analysis of nanomorphologies in advanced energy materials. His personal website can be found under http://www.uni-ulm.de/stochastik. His email address is ole.stenzel@uni-ulm.de

Björn Baumeier is a Postdoctoral Research Fellow in Denis Andrienko's group at the Max Planck Institute for Polymer Research. His work focuses primarily on first-principles calculations of ground and excited state properties of molecular structures relevant for transport simulations. He obtained his Diploma and $\mathrm{PhD}$ in solid state physics from the University of Münster, Germany, working in the group of Prof. J. Pollmann on self-interaction corrections to density-functional theory, and has worked on surface plasmonics with Prof. A. Maradudin at the University of California, Irvine. His website is http://www.mpip-mainz.mpg.de/ baumeier/. His email address is baumeier@mpip-mainz.mpg.de

Dirk Kroese is ARC Professorial Fellow at the University of Queensland. His research interests include Monte Carlo methods, adaptive importance sampling, randomized optimization, and rare-event simulation. He has over 70 peer-reviewed publications, including three monographs: Simulation and the Monte Carlo Method, 2nd Edition, 2007, John Wiley \& Sons (with R.Y. Rubinstein), The Cross-Entropy Method, 2004, Springer-Verlag, (with R.Y. Rubinstein), and the Handbook of Monte Carlo Methods, 2011, John Wiley \& Sons (with T. Taimre and Z.I. Botev). His website is http://www.maths.uq.edu.au/ kroese. His email address is kroese@maths.uq.edu.au

Volker Schmidt is Professor at the Faculty of Mathematics and Economics of Ulm University. His research interests include stochastic geometry, spatial statistics, and Monte Carlo simulation of spatial stochastic models as well as their applications to structural analysis of (microscopic and geographically mapped) image data. He is (co-) author of more than 100 peer-reviewed publications, including several textbooks and monographs. His personal website can be found under http://www.uni-ulm.de/stochastik. His email address is volker.schmidt@uni-ulm.de 